\title{
sciendo
}

\section{The Factors that Influence Postpaid Subscribers to Make Churn in a Romanian Telecommunication Company}

\author{
Andreea DUMITRACHE \\ Bucharest University of Economic Studies, Bucharest, Romania \\ dumitrache.andreea03@gmail.com \\ Monica Mihaela MATEI MAER \\ Bucharest University of Economic Studies, Bucharest, Romania \\ matei.monicamihaela@gmail.com \\ Stelian STANCU \\ Bucharest University of Economic Studies, Bucharest, Romania \\ stelianstancu@yahoo.com \\ Oana POPESCU \\ Bucharest University of Economic Studies, Bucharest, Romania \\ predescu oana85@yahoo.com
}

\begin{abstract}
The telecommunication industry is growing every day, increasing its competitiveness. In almost all European countries, the market penetration of mobile network users exceeded 100\% (for example in Croatia it is over 130\%). Acquiring new users is virtually impossible because there are no new users. There are only users of rival companies who are exposed to numerous marketing campaigns carefully designed to try to win them. That's why customer retention activity and churn prevention is a necessity. The purpose of this paper is to predict customers who are willing to migrate to another Romanian mobile telecommunications company and to determine the strongest factors of influence in the consumer's decision to leave their current service provider for another provider. Migration behavior analysis is developed for customers with postpaid subscriptions. We applied the ROSE package for resampling and decision trees on the dataset to identify decision makers in the migration process. The combination of the two techniques in our study did not significantly improve the performance of the classifier measured by the AUC (Area Under the Curve). After balancing the sample, however, we obtain the optimal value of the AUC coefficient (0.724) for the second cluster, making the correct prediction of the churn phenomenon on the analyzed data set. The study is an addition of Churn Analysis in Romanian Telecommunications Company, M. M. Matei Maer and A. Dumitrache (2018), where ROSE and logistic regression was applied to the same dataset for the same purpose: balancing the sample and churn prediction, but the value of the AUC coefficient was really low, making it difficult to accurately predict the churn phenomenon. Therefore, another purpose of the current paper is to compare the performance of the two techniques used in combination with ROSE on the same set of data.
\end{abstract}

Keywords: churn, imbalance class, decisional trees, Romanian telecommunications.

\section{Introduction}

Telecommunications is one of the most dynamic industries. Businesses that are part of this 
sector have faced multiple threats of financial losses from migrant customers who want to leave their current service provider in exchange for other offers from competing companies. Businesses face aggressive competition to keep their customers and therefore require an efficient churn prediction model to monitor and streamline customers' current situation.

Maintaining existing users is extremely important because it is five to seven times cheaper to retain a consumer than to buy another one (Essam Shaaban et al., 2012). So, due to the high cost of acquiring new customers, the prediction of this action in the telecommunications sector has become an indispensable part of the whole business process of a business.

The purpose of this paper is to anticipate customers who are willing to migrate to another Romanian mobile telecommunications company and to determine the strongest factors of influence in the consumer's decisions. This study complements the paper written by Maer Matei M. M. \& Dumitrache A. (2018) and seeks to test the compatibility and efficiency of the ROSE junction with decision trees, on the same set of data used and in the reference work. The analysis of migratory behavior, found in the literature as churn, is developed for postpaid customers.

The churn phenomenon has taken a major role in the Romanian telecommunications industry according to the ANCOM portability report (http://www.ancom.org.ro/), which states that over a decade after the launch of the portability service, more than 5.1 million telephone numbers have been ported, nearly 4.4 million of which are mobile numbers and over 700,000 fixed telephony numbers. This portability service allows consumers to keep their phone number when changing service providers, thus increasing the freedom of migration from one supplier to another and giving them the opportunity to enjoy the benefits of a competitive market. Since October 2008, when the service was launched on the market, and by the middle of October 2018, a total of 5,131,415 numbers were ported, of which 4,391,304 are mobile numbers.

At the end of the twentieth century, scientists focused on theoretical forecasting models for improving and upgrading algorithms and data extraction methods. However, in the twenty-one century, the focus is on the scientific research of data mining and predictive modeling techniques and their application in the real environment (Michael J. A. Berry \& Gordon S. Linoff, 2004). The number of studies published in recent years on churn predictions in telecommunications proves that this issue has become a major concern. So far, the publication of the themes based on the predictive modeling of this phenomenon on the Romanian data sets is still at the beginning.

\section{Literature review}

Increasingly more works have focused on the following data mining techniques: logistic regression, decision trees, random forest, neural networks, vector support machines and hybrid methods. Also, the most optimal predictive and classification models are those in which historical data was used. Few of the thousands of researchers have integrated realtime or near-real-time analyzes to reveal the prediction of customer migration (Turiho1 J.C., Cheruiyot W., Kibe1 A. \& Mungwarakarama I., 2017).

Decision trees has proved to be a very intensive classification model used to determine the most predictive factors in the churn action because the expressed results are very easy to view and interpret, each sub-tree in its composition representing a response to a classification question. Those have demonstrated their applicability to predict churn 
behavior in several countries, Egypt being one of them. The study was conducted on a data set of 5,000 records, where 22 attributes were defined, defining five categories of information: customer behavior in the company, customer satisfaction, profitability, cumulated sales in the last 3 months and demographics. Of the three modern predictive techniques applied to the dataset described above, the decision trees had an accuracy rate of 97.9\%, SVM of 83.6\%, and neural networks of 84.1\% (Essam Shaaban \& Aboul Ella Hassanien, 2016).

Exploring key indicators that drive customer migration from a telecom provider to another competing furnisher is an extremely important step in the entire churn prediction process. The behavioral analysis of customers of a telecommunications company in Pakistan suggests that the main decision maker in a customer's churn action is the price, followed by qualitative factors such as network power, roaming functionality and so on (Akamal M., 2017).

The decision-makers that provoke the churn action of customers in Bangkok, Thailand's mobile telecommunication services industry are the demographic profiles of customers and a mix of marketing factors. Hypotheses was tested using the T and ANOVA Test. The results of their implementation revealed that there are relationships between demographic profiles and marketing factors (Thamsaranasakul N., 2007).

The customer migration action towards other competing suppliers in the telecommunications industry is related to the company's life cycle on the market. If the industry is growing, then more and more customers are associated with the company. So, even if some customers are churning, the total number of clients of the company they are migrating from, continues to grow, which means that one of the decision-makers in the process may be the telecom market itself (Lejeune M., 2001).

In the context of telecommunication prediction, churn is a rare event. In all datasets, the number of churners is much higher than that of non-churners. All parametric or nonparametric classifiers are compromised by unbalanced data. In order to overcome the shortcomings encountered in the classification of instructors on unbalanced samples, a solution based on a bootstrap technique called ROSE (Random Over Sampling Examples) and as previously demonstrated in other studies, in the context of telecommunication does not improve the performance of the logistics classifier. Therefore, even after balancing the sample, an even lower value of the AUC coefficient was obtained, making it difficult to correctly predict the churn phenomenon.

\section{Methodology}

In this article we analyze churn behavior on a sample of 1,0701 randomly selected subscribers from a database of a large telecoms company operating on the Romanian market. Migrated customers are marked with 1 in the Churn variable. We can classify the variables into four major categories: demographics, where we have age information (Age), details about the customer's lifecycle in the company: his age using the company's services expressed in months (Tenure), the number of months since the last offer change (MonthsO), data on the financial strength of each customer, information expressed on the average threemonth invoice that each customer has to pay, expressed in euro (Invoice), the amount of the surcharge paid for off-site services (ExtraCosts), and information on subscriber interaction with concurrent network customers: sum of national minutes received (MinR).

The dataset analyzed describes a situation of imbalanced class, which means that the 
churners class is rare over the sample. In this paper we will use a solution based on a smooth bootstrap technique called ROSE (Random Over Sampling Examples). To balance the class distribution, new artificial data is extracted from the two kernel density estimates, as in the reference paper (Maer Matei M. M. \& Dumitrache A., 2018). We have chosen this approach because it has demonstrated excellent performance compared to other existing methods and also provides useful tools for the model evaluation phase (Cabrera, A. F., 1994).

Due to the defective distributions, decision trees will not be applied to the entire data set, as in logistic regression (Maer Matei M. M. \& Dumitrache A., 2018), it will be divided into groups as homogeneous as possible to analyze individual behavior, depending on the cluster of which it is part. We will study the three groups classified by k-means as in the previous paper. Decision trees generate output with easy marketing interpretation and easy identification of significant variables in managerial decision making. So, we will continue to generate three outputs, each assigned to a group.

\section{Results and discussions}

The first cluster decision tree (Figure 1) contains nine nodes. Of all the features involved in the model, it classifies the MonthsO and MinR variables as those with the highest importance in the classification and estimation of the churn pattern.

Therefore, the time-measured in the number of months since the last change on the account is the most predictive power factor, which influences $88 \%$ of the churn process for the first cluster, and $12 \%$ this action is influenced by the minutes received from other national networks competing with the telecommunication service provider for the individuals whose behavior is being studied.

Table 1. Importance of variables in the first cluster model

\begin{tabular}{cc}
\hline \multicolumn{2}{c}{ Variable importance } \\
\hline MonthsO & $\mathrm{MinR}$ \\
88 & 12 \\
\hline
\end{tabular}

Source: Authors' own research.

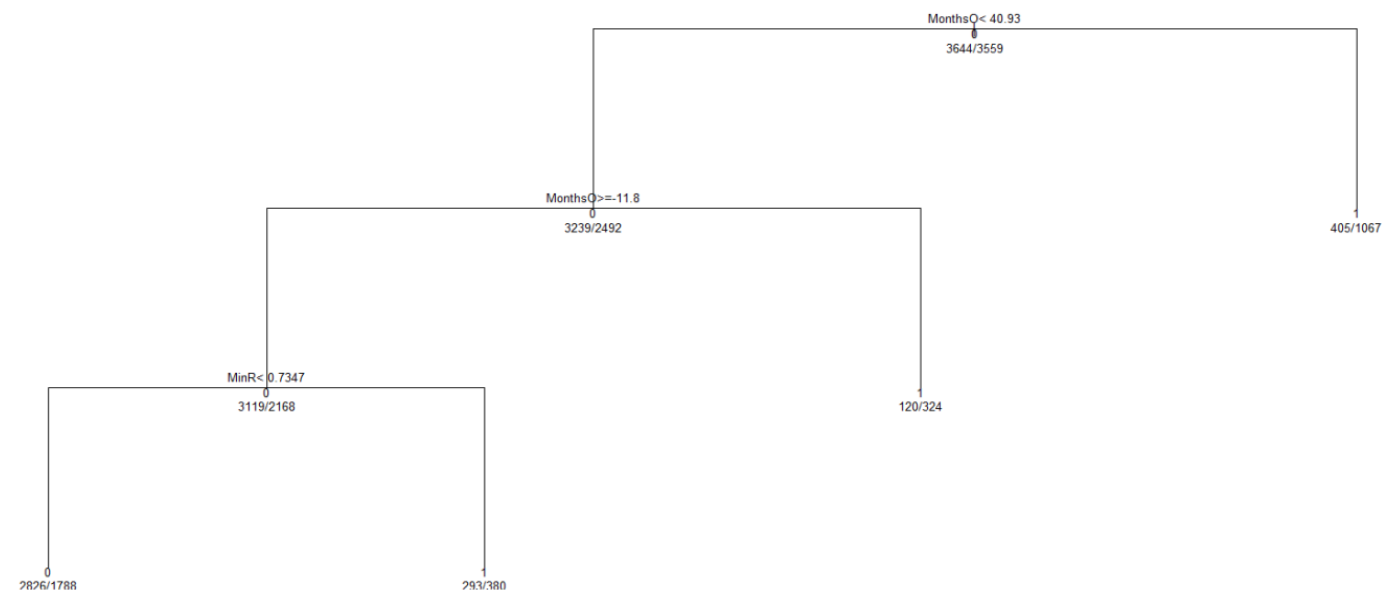

Figure 1. The first cluster decision tree

Source: Authors' own research.

The decision tree in the above figure (Figure 1) places that first decision point number 
of months since the last bid (since it changed the last bid) in the action of churn as follows:

- If it has been more than three years and four months, and the customer has not been contacted by the company to change its offer, and virtually his latest service has been renewed about 40 months ago, this customer is most prone to churn . Of the 1,472 customers presenting this risk, 1,067 were classified correctly.

- Another risk of making churn is predicted by the decision tree and if the number of months since the change of the last offer on the account is still lower than the first decision point, but is greater than 11.8 months in the closeness of one year's fulfillment. In this situation, few individuals in the dataset are classified - 444. Of these, only 324 of them have been classified correctly.

- The last case in which customers are predisposed to churn is that individuals with whom they communicate through phone calls are customers of competing companies on the market. Thus, if the number of national minutes received by a customer represents more than $73.47 \%$ of the total national minutes consumed, it will act as a churner, according to the generated decision tree. Over $75 \%$ of these individuals were classified correctly.

The AUC for this model is 0.645 . Therefore, even by balancing the sample with ROSE, we still get a reduced AUC coefficient, making it difficult to accurately predict the churn phenomenon for the first cluster. Its value is, however, comparable to that estimated by the logistic regression (Maer Matei M. M. \& Dumitrache A., 2018).

Regarding the second generated tree, which expresses the classification and estimation of the most predictive factors in the second cluster, the AUC value is an optimal one - 0.724, which means that the prediction can be interpreted as being correct, to be implemented in production because the value of the coefficient exceeds the threshold of $70 \%$ accuracy.

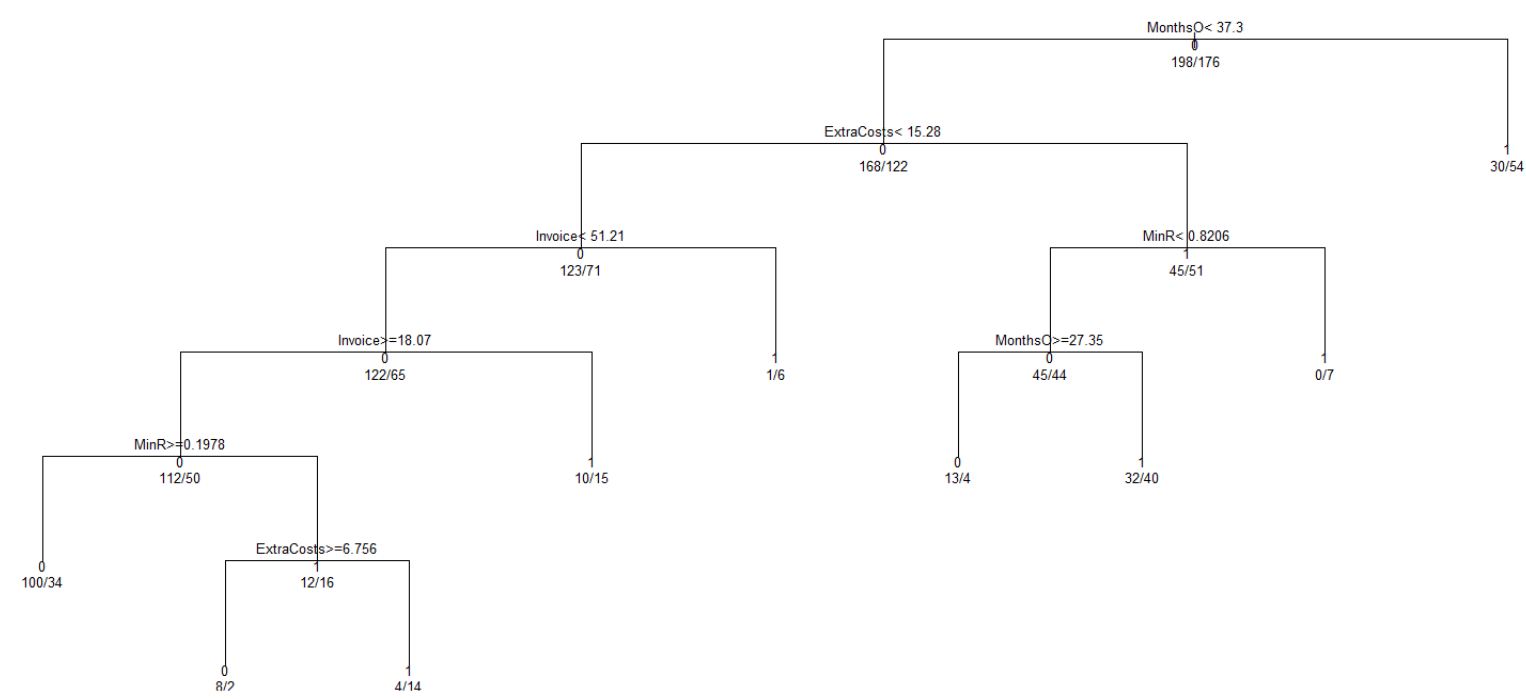

Figure 2. The decision tree of the second cluster

Source: Authors' own research.

As with the first cluster estimate, the second group decision tree (Figure 2) considers that the main decision maker in the churn action is MonthsO - the number of months since the subscription change. What is different from the first group is the multitude of predictive 
factors that influence the churn action. In this cluster we encounter four estimated variables that have predictive power in the following proportions:

$>29 \%$ represents the importance of the number of months since the offer change (MonthsO) in terms of churn;

$>22 \%$ is the amount of the invoice paid (Invoice);

$>21 \%$ are the national minutes received (MinR);

$>21 \%$ additional cost (ExtraCosts);

$>5 \%$ of his age as a client in the company (Tenure);

$>2 \%$ represents customer age (Age).

Table 2. Importance of variables in the model for the first cluster

\begin{tabular}{cccccc}
\hline \multicolumn{6}{c}{ Variable importance } \\
\hline Monthso & Invoice & MinR & ExtraCosts & Tenure & Age \\
29 & 22 & 21 & 21 & 5 & 2 \\
\hline
\end{tabular}

The decision tree in Figure 2 places the number of months since the change in the last subscription as the first decision point, as in the case of the first class, but this time very little is the starting point. The main cause of the churn is whether the customer would have had more than 37.3 months after the last offer. Over $65 \%$ of these individuals were classified correctly.

As I said above, another predictive factor, with a rather high importance in the model, is the additional cost, but also the percentage of national minutes received. If the individual exceeds the threshold of $€ 15.28$ extra cost and has incoming minutes of more than $82.06 \%$, then the decision tree classifies him as a chaperon. The individuals in this estimate were correctly classified as $100 \%$ (0/7). If, however, the customer has fewer minutes received than the above threshold and has less than 27.35 months from changing his subscription or offer, he may be classified as a non-churner, however, the accuracy of the classification in this case is very low $-23.5 \%$.

Another important feature in the churn model is the invoice value. Churners are ranked according to this factor after several thresholds of their value.

If the invoice value is higher than 51.21 euro, even if the number of months after the subscription is changed and the additional cost is below the limit above (37.3 and 15.28) then individuals are prone to churn.

However, customers are prone to make churn even if the invoice has a value below 18.07 euros, according to the decision tree. There are also two exceptions: when the percentage of national minutes received is less than $19.78 \%$ and when the additional cost is less than 6.75 euros.

Thus, as in the case of estimating and classifying predictive factors using the first cluster decision tree method, here the "rule of the game" of the churn model is given by the lifetime of the subscription and the percentage of minutes received from other national networks. Besides these two indicators, the value of the bill and the additional cost plays a very important role in the model. 


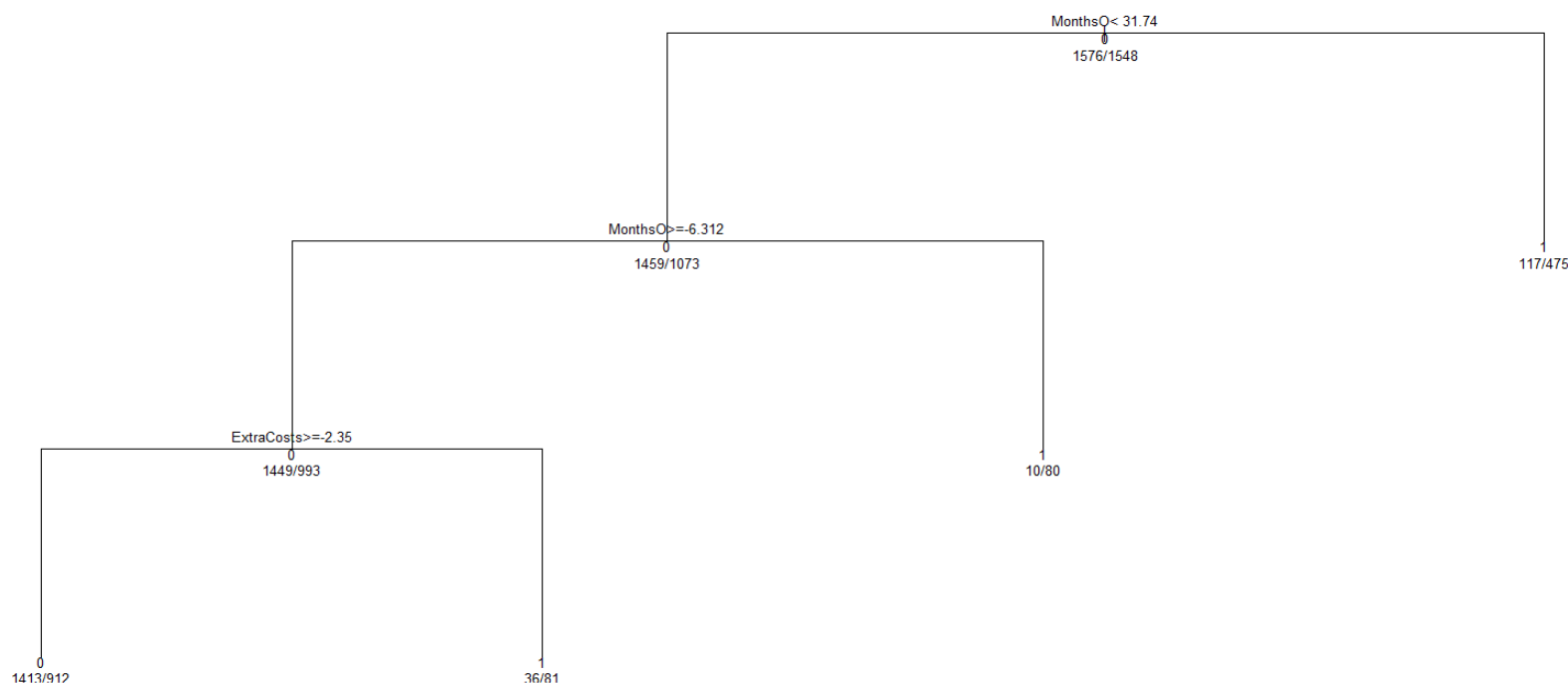

Figure 3. Decision tree for the third cluster Source: Authors' own research.

The AUC for the third cluster is 0.657 , a value comparable to that generated by the logistic regression (Maer Matei M. M. \& Dumitrache A., 2018), after balancing the sample with ROSE. As with the first cluster, the correct prediction of the cluster phenomenon for the cluster three is somewhat difficult because AUC has a low value.

The decision tree corresponding to the third cluster (Figure 3) contains five nodes. Of all the features involved, it identifies Months0 (90\%) and ExtraCosts (10\%) as the most important variables in churn prediction.

Table 3. Importance of variables in model for cluster three

\begin{tabular}{cc}
\hline \multicolumn{2}{c}{ Variable importance } \\
\hline MonthsO & ExtraCosts \\
90 & 10 \\
\hline
\end{tabular}

Source: Authors' own research

According to the decision tree (Figure 3), the first and most important decision maker is the lifetime of the last subscription change in the churn action as follows: If the number of months since the last bid change is greater than 31.74 then the individual concerned is predisposed to churn - $80 \%$ of individuals in this situation was classified correctly. It also happens if it has more than 6.31 months, but an additional cost of more than 2.35 euros. There are, however, 80 individuals out of a total of 90 as being prone to churn even if they have a number of months since the last offer change of less than 6.31 months.

\section{Conclusions}

On this set of unbalanced data, ROSE was applied - a solution based on a bootstrap technique along with a data export technique widely used in predictive modeling: decision trees. As previously demonstrated in other studies, in the context of telecommunications, sampling does not significantly improve the performance of the classifier, but in this case, after 
balancing the sample, we obtain for one of the three groups an optimal AUC coefficient, which makes it possible to accurately predict the phenomenon of churn. We can also conclude by comparing the current output with the output of the reference work (Maer Matei M. M. \& Dumitrache A., 2018) that the logistic regression is more sensitive to the imbalance class than the decision trees because in the first case none of the three clusters had an optimal value of the reference coefficient.

The decision trees applied on the re-sampled data set out the MonthsO variable - the number of months since the last offer was changed as the predictive factor with the greatest importance in the churn model, classifying it as the first decision point in all three groups. The number of months since the last offer was changed, and then the number of national minutes received, the additional cost and the invoice value are the main features that influence the churn action. Therefore, the decision makers from Romanian telecommunication sector should build their strategies to retain their clients, mainly on tools based on the optimal selection of the periodicity of updating the contract terms. Moreover, the findings of this paper emphasize other factors that should represent key indicators in the company dashboards designed to predict the risk of losing customers, such as: the interaction with competing companies and the consumption behavior.

\section{References}

Akamal M., Factor Causing Customer Churn: A Qualitative Explanation Of Customer Churns In Pakistan, Capital University of Science \& Tehnology, Islamabad, 2017.

Cabrera, A. F. (1994). Logistic regression analysis in higher education: An applied perspective, Higher Education: Handbook of Theory and Research, Vol. 10, 225-256.

Essam Shaaban, Aboul Ella Hassanien, Churn Prediction Retention Framework, International Journal of Advances in Computer Science \& Its Applications, Volume 6 : Issue 1, ISSN 2250 3765, 2016.

Essam Shaaban, Yehia Helmy, Ayman Khedr, Mona Nasr, A Proposed Churn Prediction Model, International Journal of Engineering Research and Applications (IJERA), 2012.

Lejeune M., Measuring the impact of data mining on Churn Management, 2001.

Maer Matei M. M., Dumitrache A., Churn analysis in a Romanian telecommunications company, The 17th International Conference on Informatics in Economy Education, Research and Business Technologies, Iași, România, 17-20 Mai, http://www.conferenceie.ase.ro/ .

Michael J. A. Berry, Gordon S. Linoff, Data Mining Techniques: For Marketing, Sales and Customer Relationship Management, Wiley Publishing, Indidana, 2004.

Thamsaranasakul N., Factors that influence customer churn in telecommunication industry in Bangkok, Doctoral dissertation, SIU PS SOMMBA, 2007-03.

Turiho1 J.C., Cheruiyot W., Kibe1 A. și Mungwarakarama I., Survey of Data Mining Techniques Used for Real Time Churn Prediction, 2017.

http://www.ancom.org.ro/ 